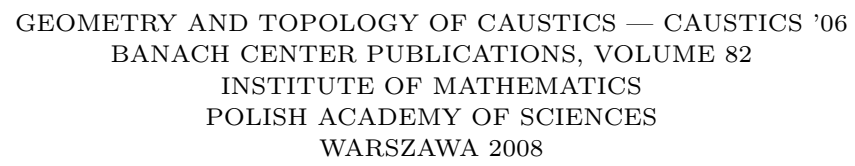

\title{
CAUSTICS AND WAVE FRONT PROPAGATIONS: APPLICATIONS TO DIFFERENTIAL GEOMETRY
}

\author{
SHYUICHI IZUMIYA \\ Department of Mathematics, Faculty of Science \\ Hokkaido University, Sapporo 060-0810, Japan \\ E-mail: izumiya@math.sci.hokudai.ac.jp \\ MASATOMO TAKAHASHI \\ Department of Mathematical Science, Common Subject Division \\ Muroran Institute of Technology, Muroran 050-8585, Japan \\ E-mail:masatomo@mmm.muroran-it.ac.jp
}

\begin{abstract}
This is mainly a survey on the theory of caustics and wave front propagations with applications to differential geometry of hypersurfaces in Euclidean space. We give a brief review of the general theory of caustics and wave front propagations, which are well-known now. We also consider a relationship between caustics and wave front propagations which might be new. Moreover, we apply this theory to differential geometry of hypersurfaces, getting new geometric properties.
\end{abstract}

1. Introduction. For a hypersurface in Euclidean space, its parallels consist of those hypersurfaces a fixed distance $r$ down the normals in a fixed direction. They always have Legendrian singularities. It is well-known that the singularities of the parallels lie on the evolute of the hypersurface. The evolute is one of the examples of caustics and the family of parallels is one of the examples of wave front propagations (also, graphlike wave fronts [9]). In this paper, we consider a relationship between the caustics and the wave front propagations. The caustics is the set of critical values of the projection a Lagrangian submanifold from the phase space onto the configuration space. In the real world, the caustics given by the reflected ray are visible. However, the wave front propagations are not visible (cf. Fig. 1). Therefore, we can say that there are hidden structures (i.e., wave front propagations) on the picture of caustics. We can draw both the parallels (i.e., wave

2000 Mathematics Subject Classification: Primary 57R45; Secondary 58Kxx.

Key words and phrases: caustics, wave fronts, evolutes, parallels, Lagrangian singularities, Legendrian singularities.

The paper is in final form and no version of it will be published elsewhere. 


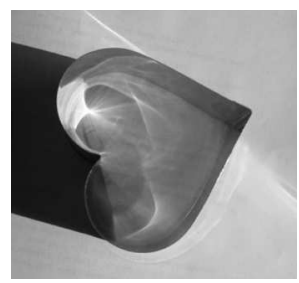

Fig. 1. The caustics reflected by a heart shape

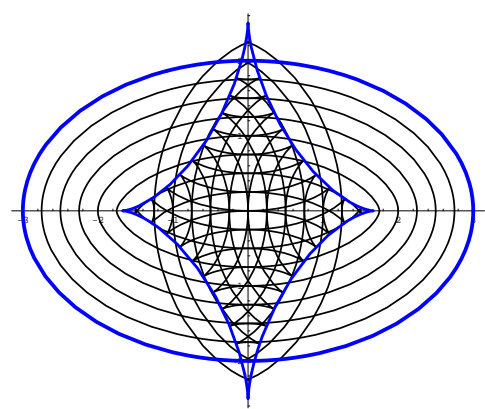

Fig. 2. The parallels and the evolute of an ellipse

front propagations) and the evolute (i.e., caustics) of an ellipse in the plane, see Fig. 2. In this picture, we can observe the relation between the evolute and the parallels. One of the main purposes in this paper is to clarify such the relation.

In $\S 2$, we give a brief review on the theory of Lagrangian singularities. The big wave front consists of a one-parameter family of wave fronts which given by the projection of a big Legendrian submanifold of the contact fibering onto the basis of this fibering. We also give a brief review on the theory of big Legendrian submanifolds in $\S 3$. We consider a special class of the big Legendrian submanifold which is called a graphlike Legendrian unfolding in [9]. The graphlike Legendrian unfolding can be always induced by Lagrangian submanifolds (cf. §4). By definition, a Lagrangian equivalence preserves the caustics. However, the converse does not hold in general even if Lagrangian submanifold germs are Lagrange stable (cf. $[1,2,22]$ ). We define the $S . P^{+}$-Legendrian equivalence among big Legendrian submanifold germs in $\S 3$. The $S . P^{+}$-Legendrian equivalence has been introduced in $[10,12,20,23]$. It preserves both the diffeomorphism types of bifurcations for families of small fronts (i.e., wave front propagations) and the caustics. In [15], it has been shown that Lagrangian equivalence implies the $S . P^{+}$-Legendrian equivalence of the induced graphlike Legendrian unfoldings from Lagrangian submanifolds (cf. Proposition 4.2 ), so that it preserves the diffeomorphism types of bifurcations wave front propagations. One of the main results in $\S 4$ is that the converse holds when Lagrangian submanifold germs are Lagrange stable (Theorem 4.6).

As an application, we investigate a relationship between the evolute and the family of parallels of a hypersurface in Euclidean space by using distance squared functions. We show that the distance squared function is a Morse family of functions and the graphlike distance squared function is a big Morse family of hypersurfaces in $\S 5$. We also review 
the theory of contact between submanifolds due to Montaldi [18] in $\S 7$ as the appendix. In [15], we have considered the contact of hypersurfaces with one-parameter family of hypersurfaces in Minkowski pseudo-hyperspheres as in Montaldi's result. We have also considered the contact of hypersurfaces with a family of hyperspheres in hyperbolic space $[13,14]$. This technique is useful for the study of the contact of hypersurfaces with families of hyperspheres in Euclidean space in $\S 6$.

We shall assume throughout the whole paper that all maps and manifolds are $C^{\infty}$ unless the contrary is explicitly stated.

2. Lagrangian submanifolds and caustics. In this section, we give a brief review on the theory of Lagrangian singularities due to [1] (also see $[2,3,5,8,11,21,22,23]$ ). We consider the cotangent bundle $\pi: T^{*} \mathbb{R}^{n} \rightarrow \mathbb{R}^{n}$ over $\mathbb{R}^{n}$. Let $(x, p)=\left(x_{1}, \ldots, x_{n}, p_{1}, \ldots, p_{n}\right)$ be the canonical coordinate on $T^{*} \mathbb{R}^{n}$. Then the canonical symplectic structure on $T^{*} \mathbb{R}^{n}$ is given by the canonical two form $\omega=\sum_{i=1}^{n} d p_{i} \wedge d x_{i}$. A submanifold $i: L \subset T^{*} \mathbb{R}^{n}$ is a Lagrangian submanifold if $\operatorname{dim} L$ and $i^{*} \omega=0$. In this case, the critical value of $\pi \circ i$ is called the caustic of $i: L \subset T^{*} \mathbb{R}^{n}$ and it is denoted by $C_{L}$. The main result in the theory of Lagrangian singularities is to describe Lagrangian submanifold germs by using families of function germs. Let $F:\left(\mathbb{R}^{k} \times \mathbb{R}^{n}, 0\right) \rightarrow(\mathbb{R}, 0)$ be an $n$-parameter unfolding of function germs. We say that $F$ is a Morse family of functions if the map germ

$$
\Delta F=\left(\frac{\partial F}{\partial q_{1}}, \ldots, \frac{\partial F}{\partial q_{k}}\right):\left(\mathbb{R}^{k} \times \mathbb{R}^{n}, 0\right) \rightarrow\left(\mathbb{R}^{k}, 0\right)
$$

is a non-singular, where $(q, x)=\left(q_{1}, \ldots, q_{k}, x_{1}, \ldots, x_{n}\right) \in\left(\mathbb{R}^{k} \times \mathbb{R}^{n}, 0\right)$. In this case, we have a smooth $n$-dimensional submanifold germ $C(F)=(\Delta F)^{-1}(0) \subset\left(\mathbb{R}^{k} \times \mathbb{R}^{n}, 0\right)$ and a map germ $L(F):(C(F), 0) \rightarrow T^{*} \mathbb{R}^{n}$ defined by

$$
L(F)(q, x)=\left(x, \frac{\partial F}{\partial x_{1}}(q, x), \ldots, \frac{\partial F}{\partial x_{n}}(q, x)\right) .
$$

We can show that $L(F)(C(F))$ is a Lagrangian submanifold germ. Then we have the following fundamental result ([1], page 300).

THEOREM 2.1. All Lagrangian submanifold germs in $T^{*} \mathbb{R}^{n}$ are constructed by the above method.

For an $n$-parameter unfolding of function germs $F:\left(\mathbb{R}^{k} \times \mathbb{R}^{n}, 0\right) \rightarrow(\mathbb{R}, 0)$, we call

$$
C(F)=\left\{(q, x) \in\left(\mathbb{R}^{k} \times \mathbb{R}^{n}, 0\right) \mid \frac{\partial F}{\partial q_{1}}(q, x)=\cdots=\frac{\partial F}{\partial q_{k}}(q, x)=0\right\}
$$

the catastrophe set of $F$, and

$$
\begin{aligned}
\mathcal{B}_{F}=\left\{x \in\left(\mathbb{R}^{n}, 0\right) \mid \text { there exists } q \in\left(\mathbb{R}^{k}, 0\right)\right. \text { such that } \\
\left.\qquad(q, x) \in C(F), \operatorname{rank}\left(\frac{\partial^{2} F}{\partial q_{i} \partial q_{j}}(q, x)\right)<k\right\}
\end{aligned}
$$

the bifurcation set of $F$. We also call $F$ a generating family of $L(F)(C(F))$. Let $\pi_{n}$ : $\left(\mathbb{R}^{k} \times \mathbb{R}^{n}, 0\right) \rightarrow\left(\mathbb{R}^{n}, 0\right)$ be the canonical projection, then we can easily show that the 
bifurcation set of $F$ is the critical value set of $\left.\pi_{n}\right|_{C(F)}$. Hence, we observe that the caustic of $L(F)$ coincides with the bifurcation set of $F$, namely, $C_{L(F)(C(F))}=\mathcal{B}_{F}$.

We now define an equivalence relation among Lagrangian submanifold germs. Let $i:(L, x) \subset\left(T^{*} \mathbb{R}^{n}, p\right)$ and $i^{\prime}:\left(L^{\prime}, x^{\prime}\right) \subset\left(T^{*} \mathbb{R}^{n}, p^{\prime}\right)$ be Lagrangian submanifold germs. Then we say that $i$ and $i^{\prime}$ are Lagrangian equivalent if there exist a diffeomorphism germ $\sigma:(L, x) \rightarrow\left(L^{\prime}, x^{\prime}\right)$, a symplectic diffeomorphism germ $\hat{\tau}:\left(T^{*} \mathbb{R}^{n}, p\right) \rightarrow\left(T^{*} \mathbb{R}^{n}, p^{\prime}\right)$ and a diffeomorphism germ $\tau:\left(\mathbb{R}^{n}, \pi(p)\right) \rightarrow\left(\mathbb{R}^{n}, \pi\left(p^{\prime}\right)\right)$ such that $\hat{\tau} \circ i=i^{\prime} \circ \sigma$ and $\pi \circ \hat{\tau}=\tau \circ \pi$, where $\pi:\left(T^{*} \mathbb{R}^{n}, p\right) \rightarrow\left(\mathbb{R}^{n}, \pi(p)\right)$ is the canonical projection and a symplectic diffeomorphism germ is a diffeomorphism germ which preserves symplectic structure on $T^{*} \mathbb{R}^{n}$. In this case, the caustic $C_{L}$ is diffeomorphic to the caustic $C_{L^{\prime}}$ by the diffeomorphism germ $\tau$.

A Lagrangian submanifold germ in $T^{*} \mathbb{R}^{n}$ at a point is said to be Lagrange stable if for every map with the given inclusion germ there is a neighborhood in the space of Lagrangian immersions (in the Whitney $C^{\infty}$-topology) and a neighborhood of the original point such that each Lagrangian immersion belonging to the first neighborhood has in the second neighborhood a point at which its germ is Lagrangian equivalent to the original germ.

We can interpret the Lagrangian equivalence by using the notion of generating families. Let $\mathcal{E}_{x}$ be the ring of function germs of $x=\left(x_{1}, \ldots, x_{n}\right)$ variables at the origin and $\mathfrak{M}_{x}=\left\{h \in \mathcal{E}_{x} \mid h(0)=0\right\}$ be the unique maximal ideal. Let $F$ and $G:\left(\mathbb{R}^{k} \times \mathbb{R}^{n}, 0\right) \rightarrow$ $(\mathbb{R}, 0)$ be function germs. We say that $F$ and $G$ are $P-\mathcal{R}^{+}$-equivalent if there exist a diffeomorphism germ $\Phi:\left(\mathbb{R}^{k} \times \mathbb{R}^{n}, 0\right) \rightarrow\left(\mathbb{R}^{k} \times \mathbb{R}^{n}, 0\right)$ of the form $\Phi(q, x)=\left(\phi_{1}(q, x), \phi_{2}(x)\right)$ and a function germ $h:\left(\mathbb{R}^{n}, 0\right) \rightarrow \mathbb{R}$ such that $G(q, x)=F(\Phi(q, x))+h(x)$. For any $F_{1}:\left(\mathbb{R}^{k} \times \mathbb{R}^{n}, 0\right) \rightarrow(\mathbb{R}, 0)$ and $F_{2}:\left(\mathbb{R}^{k^{\prime}} \times \mathbb{R}^{n}, 0\right) \rightarrow(\mathbb{R}, 0), F_{1}$ and $F_{2}$ are said to be stably $P-\mathcal{R}^{+}$-equivalent if they become $P$ - $\mathcal{R}^{+}$-equivalent after the addition to the arguments to $q_{i}$ of new arguments $q_{i}^{\prime}$ and to the functions $F_{i}$ of nondegenerate quadratic forms $Q_{i}$ in the new arguments (i.e., $F_{1}+Q_{1}$ and $F_{2}+Q_{2}$ are $P$ - $\mathcal{R}^{+}$-equivalent).

Let $F:\left(\mathbb{R}^{k} \times \mathbb{R}^{n}, 0\right) \rightarrow(\mathbb{R}, 0)$ be a function germ. We say that $F$ is an $\mathcal{R}^{+}$-versal deformation of $f=\left.F\right|_{\mathbb{R}^{k} \times\{0\}}$ if

$$
\mathcal{E}_{q}=J_{f}+\left\langle\frac{\partial F}{\partial x_{1}}\left|\mathbb{R}^{k} \times\{0\}, \ldots, \frac{\partial F}{\partial x_{n}}\right| \mathbb{R}^{k} \times\{0\}\right\rangle_{\mathbb{R}}+\langle 1\rangle_{\mathbb{R}}
$$

where

$$
J_{f}=\left\langle\frac{\partial f}{\partial q_{1}}(q), \ldots, \frac{\partial f}{\partial q_{k}}(q)\right\rangle_{\mathcal{E}_{q}} .
$$

Then we have the following theorem:

Theorem 2.2. Let $F:\left(\mathbb{R}^{k} \times \mathbb{R}^{n}, 0\right) \rightarrow(\mathbb{R}, 0)$ and $G:\left(\mathbb{R}^{k^{\prime}} \times \mathbb{R}^{n}, 0\right) \rightarrow(\mathbb{R}, 0)$ be Morse families of functions. Then we have the following:

(1) $L(F)(C(F))$ and $L(G)(C(G))$ are Lagrangian equivalent if and only if $F$ and $G$ are stably $P$ - $\mathcal{R}^{+}$-equivalent.

(2) $L(F)(C(F))$ is Lagrange stable if and only if $F$ is an $\mathcal{R}^{+}$-versal deformation of $f=\left.F\right|_{\mathbb{R}^{k} \times\{0\}}$. 
For the proof of the above theorem, see [1], page 304 and 325. The following proposition describes the well-known relationship between bifurcation sets and equivalence among unfoldings of function germs:

Proposition 2.3. Let $F, G:\left(\mathbb{R}^{k} \times \mathbb{R}^{n}, 0\right) \rightarrow(\mathbb{R}, 0)$ be function germs. If $F$ and $G$ are $P-\mathcal{R}^{+}$-equivalent then there exists a diffeomorphism germ $\phi:\left(\mathbb{R}^{n}, 0\right) \rightarrow\left(\mathbb{R}^{n}, 0\right)$ such that $\phi\left(\mathcal{B}_{F}\right)=\mathcal{B}_{G}$.

3. Legendrian submanifolds and wave front propagations. In this section, we give a brief review of the classification theory of both the families of wave fronts and the discriminants.

We consider the projective cotangent bundle $\bar{\pi}: P T^{*}\left(\mathbb{R}^{n} \times \mathbb{R}\right) \rightarrow \mathbb{R}^{n} \times \mathbb{R}$ over $\mathbb{R}^{n} \times \mathbb{R}$. Let $\Pi: T P T^{*}\left(\mathbb{R}^{n} \times \mathbb{R}\right) \rightarrow P T^{*}\left(\mathbb{R}^{n} \times \mathbb{R}\right)$ be the tangent bundle over $P T^{*}\left(\mathbb{R}^{n} \times \mathbb{R}\right)$ and $d \bar{\pi}: T P T^{*}\left(\mathbb{R}^{n} \times \mathbb{R}\right) \rightarrow T\left(\mathbb{R}^{n} \times \mathbb{R}\right)$ the differential map of $\bar{\pi}$.

For any $X \in T P T^{*}\left(\mathbb{R}^{n} \times \mathbb{R}\right)$, there exists an element $\alpha \in T_{(x, t)}^{*}\left(\mathbb{R}^{n} \times \mathbb{R}\right)$ such that $\Pi(X)=[\alpha]$. For an element $V \in T_{(x, t)}\left(\mathbb{R}^{n} \times \mathbb{R}\right)$, the property $\alpha(V)=0$ does not depend on the choice of representative of the class $[\alpha]$. Thus we can define the canonical contact structure on $P T^{*}\left(\mathbb{R}^{n} \times \mathbb{R}\right)$ by

$$
K=\left\{X \in T P T^{*}\left(\mathbb{R}^{n} \times \mathbb{R}\right) \mid \Pi(X)(d \bar{\pi}(X))=0\right\} .
$$

Because of the trivialization $P T^{*}\left(\mathbb{R}^{n} \times \mathbb{R}\right) \cong\left(\mathbb{R}^{n} \times \mathbb{R}\right) \times P\left(\mathbb{R}^{n} \times \mathbb{R}\right)^{*}$, we call

$$
\left(\left(x_{1}, \ldots, x_{n}, t\right),\left[\xi_{1}: \cdots: \xi_{n}: \tau\right]\right)
$$

a homogeneous coordinate, where $\left[\xi_{1}: \cdots: \xi_{n}: \tau\right]$ is the homogeneous coordinate of the dual projective space $P\left(\mathbb{R}^{n} \times \mathbb{R}\right)^{*}$. It is easy to show that $X \in K_{((x, t),[\xi: \tau])}$ if and only if $\sum_{i=1}^{n} \mu_{i} \xi_{i}+\lambda \tau=0$, where $d \bar{\pi}(X)=\sum_{i=1}^{n} \mu_{i}\left(\partial / \partial x_{i}\right)+\lambda(\partial / \partial t)$.

We remark that $P T^{*}\left(\mathbb{R}^{n} \times \mathbb{R}\right)$ is a fiberwise compactification of the 1 -jet space $J^{1}\left(\mathbb{R}^{n}, \mathbb{R}\right)$ as follows: We consider an affine open subset $U_{\tau}=\{((x, t),[\xi: \tau]) \mid \tau \neq 0\}$ of $P T^{*}\left(\mathbb{R}^{n} \times \mathbb{R}\right)$. For any $((x, t),[\xi: \tau]) \in U_{\tau}$, we have

$$
\left(\left(x_{1}, \ldots, x_{n}, t\right),\left[\xi_{1}: \cdots: \xi_{n}: \tau\right]\right)=\left(\left(x_{1}, \ldots, x_{n}, t\right),\left[-\left(\xi_{1} / \tau\right): \cdots:-\left(\xi_{n} / \tau\right):-1\right]\right),
$$

so that we may adopt the corresponding affine coordinates $\left(\left(x_{1}, \ldots, x_{n}, t\right),\left(p_{1}, \ldots, p_{n}\right)\right)$, where $p_{i}=-\xi_{i} / \tau$. On $U_{\tau}$ we can easily show that $\theta^{-1}(0)=K \mid U_{\tau}$, where $\theta=d t-$ $\sum_{i=1}^{n} p_{i} d x_{i}$. This means that $U_{\tau}$ may be identified with the 1 -jet space $J^{1}\left(\mathbb{R}^{n}, \mathbb{R}\right)$. We call the above coordinate a system of canonical coordinates. Throughout the remainder of this paper, we use this identification so that we have $J^{1}\left(\mathbb{R}^{n}, \mathbb{R}\right) \subset P T^{*}\left(\mathbb{R}^{n} \times \mathbb{R}\right)$.

A submanifold $i: L \subset P T^{*}\left(\mathbb{R}^{n} \times \mathbb{R}\right)$ is a Legendrian submanifold if $\operatorname{dim} L$ and $d i_{p}\left(T_{p} L\right) \subset K_{i(p)}$ for any $p \in L$. We say that a point $p \in L$ is a Legendrian singular point if $\operatorname{rank} d(\bar{\pi} \circ i)_{p}<n$.

For a Legendrian submanifold $i: L \subset P T^{*}\left(\mathbb{R}^{n} \times \mathbb{R}\right), \bar{\pi} \circ i(L)=W(L)$ is called a big wave front. We have a family of small fronts:

$$
W_{t}(L)=\pi_{1}\left(\pi_{2}^{-1}(t) \cap W(L)\right) \quad(t \in \mathbb{R}),
$$

where $\pi_{1}: \mathbb{R}^{n} \times \mathbb{R} \rightarrow \mathbb{R}^{n}$ and $\pi_{2}: \mathbb{R}^{n} \times \mathbb{R} \rightarrow \mathbb{R}$ are the canonical projections which gives $\pi_{1}(x, t)=x$ and $\pi_{2}(x, t)=t$ respectively. In this sense, we call $L$ a big Legendrian submanifold. The discriminant of the family $W_{t}(L)$ is defined as the image of singular 
points of $\pi_{1} \mid W(L)$. In the general case, the discriminant consists of three components: the caustics $C_{L}$, the projection of the set of singular points of $W(L)$, the Maxwell stratum $M_{L}$, the projection of self intersection points of $W(L)$; and also of the envelope of the family of small fronts $\Delta$ (for more detail, see $[15,23]$ ).

For any Legendrian submanifold germ $i:\left(L, p_{0}\right) \subset\left(P T^{*}\left(\mathbb{R}^{n} \times \mathbb{R}\right), p_{0}\right)$, there exists a generating family of $i$ by the theory of Legendrian singularity [1]. Let $F:\left(\mathbb{R}^{k} \times\left(\mathbb{R}^{n} \times\right.\right.$ $\mathbb{R}), 0) \rightarrow(\mathbb{R}, 0)$ be a function germ such that $\left(F, d_{2} F\right):\left(\mathbb{R}^{k} \times\left(\mathbb{R}^{n} \times \mathbb{R}\right), 0\right) \rightarrow\left(\mathbb{R} \times \mathbb{R}^{k}, 0\right)$ is a non-singular, where

$$
d_{2} F(q, x, t)=\left(\frac{\partial F}{\partial q_{1}}(q, x, t), \ldots, \frac{\partial F}{\partial q_{k}}(q, x, t)\right) .
$$

In this case, we call $F$ a big Morse family of hypersurfaces. Then $\Sigma_{*}(F)=\left(F, d_{2} F\right)^{-1}(0)$ is a smooth $n$-dimensional submanifold germ. Define

$$
\mathcal{L}_{F}:\left(\Sigma_{*}(F), 0\right) \rightarrow P T^{*}\left(\mathbb{R}^{n} \times \mathbb{R}\right)
$$

by

$$
\mathcal{L}_{F}(q, x, t)=\left(x, t,\left[\frac{\partial F}{\partial x}(q, x, t): \frac{\partial F}{\partial t}(q, x, t)\right]\right)
$$

where

$$
\left[\frac{\partial F}{\partial x}(q, x, t): \frac{\partial F}{\partial t}(q, x, t)\right]=\left[\frac{\partial F}{\partial x_{1}}(q, x, t): \cdots: \frac{\partial F}{\partial x_{n}}(q, x, t): \frac{\partial F}{\partial t}(q, x, t)\right] .
$$

It is easy to show that $\mathcal{L}_{F}\left(\Sigma_{*}(F)\right)$ is a Legendrian submanifold germ. By the main theorem of Arnol'd-Zakalyukin [1], we can show the following proposition:

Proposition 3.1. All big Legendrian submanifold germs are constructed by the above method.

For a function germ $F:\left(\mathbb{R}^{k} \times\left(\mathbb{R}^{n} \times \mathbb{R}\right), 0\right) \rightarrow(\mathbb{R}, 0)$, we call

$D(F)=\left\{(x, t) \in\left(\mathbb{R}^{n} \times \mathbb{R}, 0\right) \mid\right.$ there exists $q \in\left(\mathbb{R}^{k}, 0\right)$ such that $\left.(q, x, t) \in \Sigma_{*}(F)\right\}$ the discriminant set of $F$.

Let $F:\left(\mathbb{R}^{k} \times\left(\mathbb{R}^{n} \times \mathbb{R}\right), 0\right) \rightarrow(\mathbb{R}, 0)$ be a big Morse family of hypersurfaces. We call $F$ a generating family of $\mathcal{L}_{F}$. In this case, we observe that the big wave front coincides with the discriminant set of $F$, namely, $W\left(\mathcal{L}_{F}\left(\Sigma_{*}(F)\right)\right)=D(F)$.

We now consider an equivalence relation among Legendrian submanifolds which preserves both the qualitative pictures of bifurcations and the discriminant of families of small fronts.

Let $i:\left(L, p_{0}\right) \subset\left(P T^{*}\left(\mathbb{R}^{n} \times \mathbb{R}\right), p_{0}\right)$ and $i^{\prime}:\left(L^{\prime}, p_{0}^{\prime}\right) \subset\left(P T^{*}\left(\mathbb{R}^{n} \times \mathbb{R}\right), p_{0}^{\prime}\right)$ be Legendrian submanifold germs. We say that $i$ and $i^{\prime}$ are strictly parametrized ${ }^{+}$Legendrian equivalent (or, briefly S.P $P^{+}$-Legendrian equivalent) if there exist diffeomorphism germs $\Phi:\left(\mathbb{R}^{n} \times\right.$ $\left.\mathbb{R}, \bar{\pi}\left(p_{0}\right)\right) \rightarrow\left(\mathbb{R}^{n} \times \mathbb{R}, \bar{\pi}\left(p_{0}^{\prime}\right)\right)$ of the form $\Phi(x, t)=\left(\phi_{1}(x), t+\alpha(x)\right)$ and $\Psi:\left(L, p_{0}\right) \rightarrow$ $\left(L^{\prime}, p_{0}^{\prime}\right)$ such that $\widehat{\Phi} \circ i=i \circ \Psi$, where $\widehat{\Phi}:\left(P T^{*}\left(\mathbb{R}^{n} \times \mathbb{R}\right), p_{0}\right) \rightarrow\left(P T^{*}\left(\mathbb{R}^{n} \times \mathbb{R}\right), p_{0}^{\prime}\right)$ is the unique contact lift of $\Phi$.

We also consider the notion of stability of Legendrian submanifold germs with respect to $S . P^{+}$-Legendrian equivalence, analogous to the stability of Lagrangian submanifold germs with respect to Lagrangian equivalence in $\S 2$ (cf. [1, Part III]). 
The $S . P^{+}$-Legendrian equivalence has been introduced in $[10,12,20]$ for the study of completely integrable holonomic systems of first order partial differential equations. It has also been independently defined by Zakalyukin [23], and is called the strong spaceequivalence, in order to apply control theory. We remark that the $S P^{+}$-Legendrian equivalence relation among big Legendrian submanifold germs preserves both the diffeomorphism types of bifurcations for families of small fronts and discriminants.

We study the S.P $P^{+}$-Legendrian equivalence by using the notion of generating families of Legendrian submanifold germs.

Let $f, g:\left(\mathbb{R}^{k} \times \mathbb{R}, 0\right) \rightarrow(\mathbb{R}, 0)$ be function germs. We say that $f$ and $g$ are $S . P-\mathcal{K}$ equivalent (or, strictly $P$-K $\mathcal{K}$-equivalent) if there exists a diffeomorphism germ $\Phi:\left(\mathbb{R}^{k} \times\right.$ $\mathbb{R}, 0) \rightarrow\left(\mathbb{R}^{k} \times \mathbb{R}, 0\right)$ of the form $\Phi(q, t)=(\phi(q, t), t)$ such that $\langle f \circ \Phi\rangle_{\mathcal{E}_{(q, t)}}=\langle g\rangle_{\mathcal{E}_{(q, t)}}$.

Let $F, G:\left(\mathbb{R}^{k} \times\left(\mathbb{R}^{n} \times \mathbb{R}\right), 0\right) \rightarrow(\mathbb{R}, 0)$ be function germs. We say that $F$ and $G$ are $x$-S.P $P^{+} \mathcal{K}$-equivalent if there exists a diffeomorphism germ $\Phi:\left(\mathbb{R}^{k} \times\left(\mathbb{R}^{n} \times \mathbb{R}\right), 0\right) \rightarrow\left(\mathbb{R}^{k} \times\right.$ $\left.\left(\mathbb{R}^{n} \times \mathbb{R}\right), 0\right)$ of the form $\Phi(q, x, t)=\left(\phi(q, x, t), \phi_{1}(x), t+\alpha(x)\right)$ such that $\langle F \circ \Phi\rangle_{\mathcal{E}_{(q, x, t)}}=$ $\langle G\rangle_{\mathcal{E}_{(q, x, t)}}$.

The notion of $S . P^{+}-\mathcal{K}$-versal deformation plays an important role for our purpose. We define the extended tangent space of $f:\left(\mathbb{R}^{k} \times \mathbb{R}, 0\right) \rightarrow(\mathbb{R}, 0)$ relative to $S . P^{+}{ }_{-} \mathcal{K}$ by

$$
T_{e}\left(S . P^{+}-\mathcal{K}\right)(f)=\left\langle\frac{\partial f}{\partial q_{1}}, \ldots, \frac{\partial f}{\partial q_{k}}, f\right\rangle_{\mathcal{E}_{(q, t)}}+\left\langle\frac{\partial f}{\partial t}\right\rangle_{\mathbb{R}} .
$$

Then we say that $F$ is an $S . P^{+}{ }_{-} \mathcal{K}$-versal deformation of $f=\left.F\right|_{\mathbb{R}^{k} \times\{0\} \times \mathbb{R}}$ if it satisfies

$$
\mathcal{E}_{(q, t)}=T_{e}\left(S . P^{+}-\mathcal{K}\right)(f)+\left\langle\frac{\partial F}{\partial x_{1}}\left|\mathbb{R}^{k} \times\{0\} \times \mathbb{R}, \ldots, \frac{\partial F}{\partial x_{n}}\right| \mathbb{R}^{k} \times\{0\} \times \mathbb{R}\right\rangle_{\mathbb{R}} .
$$

We remark that $F$ is $S . P^{+}{ }_{-} \mathcal{K}$-versal, then $n$ is an upper bound for

$$
\operatorname{dim}_{\mathbb{R}} \mathcal{E}_{(q, t)} / T_{e}\left(S . P^{+}{ }_{-} \mathcal{K}\right)(f) .
$$

Moreover, we have the following very important property as a consequence of the versality theorem [4].

Proposition 3.2. (1) Let $F$ and $G$ be n-parameter $S . P^{+}{ }_{-} \mathcal{K}$-versal deformations of $f$. Then $F$ and $G$ are $x-S . P^{+}{ }_{-} \mathcal{K}$-equivalent.

(2) Let $\xi_{1}(q, t), \ldots, \xi_{n}(q, t)$ be generators of the $\mathbb{R}$-vector space $\mathcal{E}_{(q, t)} / T_{e}\left(S . P^{+}-\mathcal{K}\right)(f)$. Then any $n$-parameter $S . P^{+}{ }_{-} \mathcal{K}$-versal deformations are $x$-S.P $P^{+}{ }_{-} \mathcal{K}$-equivalent to

$$
F(q, x, t)=f(q, t)+\sum_{i=1}^{n} x_{i} \xi_{i}(q, t) .
$$

TheOREM 3.3. Let $F:\left(\mathbb{R}^{k} \times\left(\mathbb{R}^{n} \times \mathbb{R}\right), 0\right) \rightarrow(\mathbb{R}, 0)$ and $G:\left(\mathbb{R}^{k^{\prime}} \times\left(\mathbb{R}^{n} \times \mathbb{R}\right), 0\right) \rightarrow(\mathbb{R}, 0)$ be big Morse families of hypersurfaces. Then

(1) $\mathcal{L}_{F}(C(F))$ and $\mathcal{L}_{G}(C(G))$ are $S . P^{+}{ }_{-}$Legendrian equivalent if and only if $F$ and $G$ are stably $x$-S.P. $P^{+}-\mathcal{K}$-equivalent.

(2) $\mathcal{L}_{F}(C(F))$ is $S . P^{+}$-Legendre stable if and only if $F$ is a $S . P^{+}{ }_{-} \mathcal{K}$-versal deformation of $f=\left.F\right|_{\mathbb{R}^{k} \times\{0\} \times \mathbb{R}}$.

Here, $F$ and $G$ are said to be stably $x-S . P^{+}-\mathcal{K}$-equivalent if they become $x-S . P^{+}{ }_{-} \mathcal{K}$ equivalent after the addition of non-degenerate quadratic forms in additional variables $q^{\prime}$. 
Since the big Legendrian submanifold germ $i:\left(L, p_{0}\right) \subset\left(P T^{*}\left(\mathbb{R}^{n} \times \mathbb{R}\right), p_{0}\right)$ is uniquely determined on the regular part of the big wave front $W(L)$, we have the following simple but significant property of Legendrian submanifold germs:

Proposition 3.4. Let $i:\left(L, p_{0}\right) \subset\left(P T^{*}\left(\mathbb{R}^{n} \times \mathbb{R}\right), p_{0}\right)$ and $i^{\prime}:\left(L^{\prime}, p_{0}\right) \subset\left(P T^{*}\left(\mathbb{R}^{n} \times\right.\right.$ $\left.\mathbb{R}), p_{0}\right)$ be big Legendrian submanifold germs such that regular sets of $\bar{\pi} \circ i, \bar{\pi} \circ i^{\prime}$ are dense respectively. Then $\left(L, p_{0}\right)=\left(L^{\prime}, p_{0}\right)$ if and only if $\left(W(L), \bar{\pi}\left(p_{0}\right)\right)=\left(W\left(L^{\prime}\right), \bar{\pi}\left(p_{0}\right)\right)$.

This result has been firstly pointed out by Zakalyukin [22]. Also see [16]. The assumption in the above proposition is a generic condition for $i, i^{\prime}$. In particular, if $i$ and $i^{\prime}$ are $S . P^{+}$-Legendre stable, then they satisfy the assumption. Concerning the discriminant and the bifurcation of small fronts, we define the following equivalence relation among big wave front germs. Let $i:\left(L, p_{0}\right) \subset\left(P T^{*}\left(\mathbb{R}^{n} \times \mathbb{R}\right), p_{0}\right)$ and $i^{\prime}:\left(L^{\prime}, p_{0}^{\prime}\right) \subset\left(P T^{*}\left(\mathbb{R}^{n} \times \mathbb{R}\right), p_{0}^{\prime}\right)$ be big Legendrian submanifold germs. We say that $W(L)$ and $W\left(L^{\prime}\right)$ are $S . P^{+}$-diffeomorphic if there exists a diffeomorphism germ $\Phi:\left(\mathbb{R}^{n} \times \mathbb{R}, \bar{\pi}\left(p_{0}\right)\right) \rightarrow\left(\mathbb{R}^{n} \times \mathbb{R}, \bar{\pi}\left(p_{0}^{\prime}\right)\right)$ of the form $\Phi(x, t)=\left(\phi_{1}(x), t+\alpha(x)\right)$ such that $\Phi(W(L))=W\left(L^{\prime}\right)$. By Proposition 3.4, we have the following proposition.

Proposition 3.5. Let $i:\left(L, p_{0}\right) \subset\left(P T^{*}\left(\mathbb{R}^{n} \times \mathbb{R}\right), p_{0}\right)$ and $i^{\prime}:\left(L^{\prime}, p_{0}^{\prime}\right) \subset\left(P T^{*}\left(\mathbb{R}^{n} \times\right.\right.$ $\left.\mathbb{R}), p_{0}^{\prime}\right)$ be big Legendrian submanifold germs such that regular sets of $\bar{\pi} \circ i, \bar{\pi} \circ i^{\prime}$ are dense respectively. Then $i$ and $i^{\prime}$ are S.P $P^{+}$-Legendrian equivalent if and only if $\left(W(L), \bar{\pi}\left(p_{0}\right)\right)$ and $\left(W\left(L^{\prime}\right), \bar{\pi}\left(p_{0}^{\prime}\right)\right)$ are $S . P^{+}$-diffeomorphic.

4. Graphlike Legendrian unfoldings and equivalence relations. We now consider a special class of the big Legendrian submanifolds in $J^{1}\left(\mathbb{R}^{n}, \mathbb{R}\right) \subset P T^{*}\left(\mathbb{R}^{n} \times \mathbb{R}\right)$. We say that a big Legendrian submanifold $i: L \subset J^{1}\left(\mathbb{R}^{n}, \mathbb{R}\right)$ is a graphlike Legendrian unfolding if $\pi_{2} \circ \bar{\pi} \circ i$ is a submersion at any point $p \in L$. The notion of graphlike Legendrian unfoldings has been introduced by the first named author [9] in order to describe the perestroikas of wave fronts given as the level surfaces of the solution for the eikonal equation given by a general Hamiltonian function. Since $L$ is a big Legendrian submanifold in $J^{1}\left(\mathbb{R}^{n}, \mathbb{R}\right)$, it has a generating family at least locally. In this case, it has a special form as follows: Let $\mathcal{F}:\left(\mathbb{R}^{k} \times\left(\mathbb{R}^{n} \times \mathbb{R}\right), 0\right) \rightarrow(\mathbb{R}, 0)$ be a big Morse family of hypersurfaces. We say that $\mathcal{F}$ is a graphlike Morse family of hypersurfaces if $(\partial \mathcal{F} / \partial t)(0) \neq 0$. It is easy to show that the corresponding big Legendrian submanifold germ is a graphlike Legendrian unfolding. Of course, all graphlike Legendrian unfolding germs can be constructed in the above way. We say that $\mathcal{F}$ is a graphlike generating family of $\mathcal{L}_{\mathcal{F}}\left(\Sigma_{*}(\mathcal{F})\right.$ ). However, we can reduce more strict form of graphlike generating families as follows: Let $\mathcal{F}$ be a graphlike Morse family of hypersurfaces. By the implicit function theorem, there exists a Morse family of functions $F:\left(\mathbb{R}^{k} \times \mathbb{R}^{n}, 0\right) \rightarrow(\mathbb{R}, 0)$ such that $\langle\mathcal{F}(q, x, t)\rangle_{\mathcal{E}_{(q, x, t)}}=\langle F(q, x)-t\rangle_{\mathcal{E}_{(q, x, t)}}$. Therefore $F(q, x)-t$ is a graphlike generating family of $\mathcal{L}_{\mathcal{F}}\left(\Sigma_{*}(\mathcal{F})\right)$. In this case,

$$
\Sigma_{*}(\mathcal{F})=\left\{(q, x, F(q, x)) \in\left(\mathbb{R}^{k} \times\left(\mathbb{R}^{n} \times \mathbb{R}\right), 0\right) \mid(q, x) \in C(F)\right\}
$$

and $\mathcal{L}_{\mathcal{F}}:\left(\Sigma_{*}(\mathcal{F}), 0\right) \rightarrow J^{1}\left(\mathbb{R}^{n}, \mathbb{R}\right)$ is given by

$$
\mathcal{L}_{\mathcal{F}}(q, x, F(q, x))=(L(F)(q, x), F(q, x)) \in J^{1}\left(\mathbb{R}^{n}, \mathbb{R}\right) \equiv T^{*} \mathbb{R}^{n} \times \mathbb{R} .
$$


Define a map $\mathfrak{L}_{F}:(C(F), 0) \rightarrow J^{1}\left(\mathbb{R}^{n}, \mathbb{R}\right)$ by

$$
\mathfrak{L}_{F}(q, x)=\left(x, F(q, x), \frac{\partial F}{\partial x_{1}}(q, x), \ldots, \frac{\partial F}{\partial x_{n}}(q, x)\right),
$$

then we have $\mathfrak{L}_{F}(C(F))=\mathcal{L}_{\mathcal{F}}\left(\Sigma_{*}(\mathcal{F})\right)$. We call $W\left(\mathfrak{L}_{F}\right)=\bar{\pi}\left(\mathfrak{L}_{F}(C(F))\right)$ the graphlike wave fronts of graphlike Legendrian unfolding $\mathfrak{L}_{F}$. We simply call $F$ a generating family of the graphlike Legendrian unfolding $\mathfrak{L}_{F}$.

For any Morse family of functions $F$, we denote that $\bar{F}(q, x, t)=F(q, x)-t$. Since $\bar{F}(q, x, t)$ is a big Morse family, we can use all the definitions of equivalence relations of the previous section. Moreover, we can translate the propositions and theorems into corresponding assertions in terms of graphlike Legendrian unfoldings. We also denote $\bar{f}(q, t)=f(q)-t$ for any $f \in \mathfrak{M}_{k}$. We can represent the extended tangent space of $\bar{f}:\left(\mathbb{R}^{k} \times \mathbb{R}, 0\right) \rightarrow(\mathbb{R}, 0)$ relative to $S . P^{+}-\mathcal{K}$ by

$$
T_{e}\left(S . P^{+}-\mathcal{K}\right)(\bar{f})=\left\langle\frac{\partial f}{\partial q_{1}}(q), \ldots, \frac{\partial f}{\partial q_{k}}(q), f(q)-t\right\rangle_{\mathcal{E}_{(q, t)}}+\langle 1\rangle_{\mathbb{R}} .
$$

For a deformation $\bar{F}:\left(\mathbb{R}^{k} \times \mathbb{R}^{n} \times \mathbb{R}, 0\right) \rightarrow(\mathbb{R}, 0)$ of $\bar{f}, \bar{F}$ is $S . P^{+}$- $\mathcal{K}$-versal deformation of $\bar{f}$ if and only if

$$
\mathcal{E}_{(q, t)}=T_{e}\left(S . P^{+}{ }_{-} \mathcal{K}\right)(\bar{f})+\left\langle\frac{\partial F}{\partial x_{1}}\left|\mathbb{R}^{k} \times\{0\}, \ldots, \frac{\partial F}{\partial x_{n}}\right| \mathbb{R}^{k} \times\{0\}\right\rangle_{\mathbb{R}} .
$$

Then we have the following proposition:

Proposition 4.1. Let $F:\left(\mathbb{R}^{k} \times \mathbb{R}^{n}, 0\right) \rightarrow(\mathbb{R}, 0)$ be a Morse family of functions. If $\mathfrak{L}_{F}(C(F))$ is a S.P $P^{+}$-Legendre stable, then $L(F)(C(F))$ is Lagrange stable.

Proof. Since $\mathfrak{L}_{F}(C(F))$ is $S . P^{+}$-Legendre stable, $\bar{F}$ is $S . P^{+}{ }_{-} \mathcal{K}$-versal deformation of $\bar{f}$ by Theorem 3.3. We have

$$
\mathcal{E}_{(q, t)}=T_{e}\left(S . P^{+}{ }_{-} \mathcal{K}\right)(\bar{f})+\left\langle\frac{\partial F}{\partial x_{1}}\left|\mathbb{R}^{k} \times\{0\}, \ldots, \frac{\partial F}{\partial x_{n}}\right| \mathbb{R}^{k} \times\{0\}\right\rangle_{\mathbb{R}} .
$$

It follows that there exist $\lambda_{i}, \mu \in \mathcal{E}_{(q, t)}$ and $c, c_{j} \in \mathbb{R}$ such that

$$
t=\sum_{i=1}^{k} \lambda_{i}(q, t) \frac{\partial f}{\partial q_{i}}(q)+\mu(q, t)(f(q)-t)+c+\sum_{j=1}^{n} c_{j} \frac{\partial F}{\partial x_{j}}(q, 0) .
$$

If we take $q=t=0$, then we have $0=c+\sum_{j=1}^{n} c_{j}\left(\partial F / \partial x_{j}\right)(0,0)$. On the other hand, if we take $q=0$, then we have $(\mu(0, t)+1) \cdot t=0$. It follows that $\mu(0, t) \neq 0$ at $t \neq 0$. By the continuity of the function germ $\mu$, we have $\mu(0,0) \neq 0$ and therefore $\mu(q, t) \neq 0$ around the origin. Finally if we take $t=0$, then the assertion follows from Theorem 2.2.

We now consider a relationship of the equivalence relations between Lagrangian submanifold germs and induced graphlike Legendrian unfoldings, that is, between Morse families of functions and big Morse families of graphlike Legendrian unfoldings. As a consequence, we give a relationship between caustics and graphlike wave fronts.

Proposition 4.2. If Lagrangian submanifold germs $L(F)(C(F))$ and $L(G)(C(G))$ are Lagrangian equivalent, then the induced graphlike Legendrian unfoldings $\mathfrak{L}_{F}(C(F))$ and $\mathfrak{L}_{G}(C(G))$ are S.P $P^{+}$-Legendrian equivalent. 
Proof. By Proposition 2.1, any Lagrangian submanifold germ in $T^{*} \mathbb{R}^{n}$ is given by image of $L(F)$ for a Morse family of functions $F$. Let $F$ and $G$ be Morse families of functions, then $L(F)(C(F))$ and $L(G)(C(G))$ are Lagrangian equivalent if and only if $F$ and $G$ are stably $P$ - $\mathcal{R}^{+}$-equivalent (cf. Theorem 2.2). By definition, if $F$ and $G$ are stably $P$ $\mathcal{R}^{+}$-equivalent, then $\bar{F}$ and $\bar{G}$ are stably $x-S . P^{+}-\mathcal{K}$-equivalent. By Theorem 3.3 , this completes the proof.

REMARK 4.3. The above proposition asserts that the Lagrangian equivalence is a stronger equivalence relation than the S.P $P^{+}$-Legendrian equivalence. The $S . P^{+}$-Legendrian equivalence relation among graphlike Legendrian unfoldings preserves both the diffeomorphism types of bifurcations for families of small fronts and caustics. On the other hand, if we observe the real caustics of rays, we cannot observe the structure of wave front propagations. In this sense, there are hidden structure behind the picture of real caustics. By the above proposition, the Lagrangian equivalence preserve not only the diffeomorphism type of caustics, but also the hidden geometric structure of wave front propagations.

Conversely we have the following proposition:

Proposition 4.4. Suppose that $L(F)(C(F))$ and $L(G)(C(G))$ are Lagrange stable. If $\mathfrak{L}_{F}(C(F))$ and $\mathfrak{L}_{G}(C(G))$ are $S . P^{+}$-Legendrian equivalent, then $L(F)(C(F)$ ) and $L(G)(C(G))$ are Lagrangian equivalent.

In order to show the proposition, we need the following lemma:

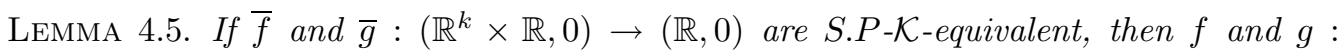
$\left(\mathbb{R}^{k}, 0\right) \rightarrow(\mathbb{R}, 0)$ are $\mathcal{R}$-equivalent, where $\bar{f}(q, t)=f(q)-t$ and $\bar{g}(q, t)=g(q)-t$.

Proof. By definition of S.P-K-K-equivalent, there exist a diffeomorphsim germ of $\Phi:\left(\mathbb{R}^{k} \times\right.$ $\mathbb{R}, 0) \rightarrow\left(\mathbb{R}^{k} \times \mathbb{R}, 0\right)$ of the form $\Phi(q, t)=(\phi(q, t), t)$ and a non-zero function germ $\lambda$ : $\left(\mathbb{R}^{k} \times \mathbb{R}, 0\right) \rightarrow \mathbb{R}$ such that $\bar{f}=\lambda \cdot \bar{g} \circ \Phi$. Then the diffeomorphism $\Phi$ preserves the zero-level set of $\bar{f}$ and $\bar{g}$, that is, $\Phi\left(\bar{f}^{-1}(0)\right)=\bar{g}^{-1}(0)$. Since the zero-level set of $\bar{f}$ is the graph of $f$ and the form of $\Phi$, we have $f=g \circ \psi$, where $\psi(q)=\phi(q, f(q))$. It is easy to show that $\psi:\left(\mathbb{R}^{k}, 0\right) \rightarrow\left(\mathbb{R}^{k}, 0\right)$ is a diffeomorphism germ. Hence $f$ and $g$ are $\mathcal{R}$-equivalent.

Proof of Proposition 4.3. By Theorem 3.3, $\bar{F}$ and $\bar{G}$ are stably $x$-S. $P^{+}-\mathcal{K}$-equivalent. It follows that $\bar{f}$ and $\bar{g}$ are stably S.P-K-K-equivalent. By Lemma 4.5, $f$ and $g$ are stably $\mathcal{R}$-equivalent. By the uniqueness of $\mathcal{R}^{+}$-versality, we have $F$ and $G$ are stably $P-\mathcal{R}^{+}$equivalent.

By definition, the set of Legendrian singular points of graphlike Legendrian unfolding $\mathfrak{L}_{F}(C(F))$ coincides with the set of singular points of $\pi \circ L(F)$. Therefore the singularities of graphlike wave fronts of $\mathfrak{L}_{F}(C(F))$ lie on the caustics of $L(F)$. Moreover, if a Lagrangian submanifold germ $L(F)(C(F))$ is a Lagrange stable, then the regular set of $\bar{\pi} \circ \mathfrak{L}_{F}(C(F))$ is dense. Hence we can apply Proposition 3.5 to our situations.

TheOREM 4.6. Suppose that $L(F)(C(F))$ and $L(G)(C(G))$ are Lagrange stable. Then the Lagrangian subamnifold germs $L(F)(C(F))$ and $L(G)(C(G)$ ) are Lagrangian equivalent if and only if the graphlike wave fronts $W\left(\mathfrak{L}_{F}\right)$ and $W\left(\mathfrak{L}_{G}\right)$ are $S . P^{+}$-diffeomorphic. 
5. Evolutes and parallels in Euclidean spaces. In this section, we firstly review the classical theory of differential geometry on hypersurfaces in Euclidean space (see, [11]). Secondly, we consider the evolutes of hypersuface as the caustics and the parallels of hypersuface as the graphlike wave fronts by using the distance squared functions (cf. $[3,19])$.

Let $\boldsymbol{x}: U \rightarrow \mathbb{R}^{n}$ be an embedding, where $U$ is an open subset in $\mathbb{R}^{n-1}$. We denote $M=\boldsymbol{x}(U)$ and identify $M$ and $U$ through the embedding $\boldsymbol{x}$. The Gauss map is defined by the unit normal vector of $M$, namely, the Gauss map $G: U \rightarrow S^{n}$ is given by $G(u)=\boldsymbol{n}(u)$, where $\boldsymbol{n}(u)$ is the unit normal vector of $M$ at $\boldsymbol{x}(u)$.

For a hypersurface $\boldsymbol{x}: U \rightarrow \mathbb{R}^{n}$, we define the evolute of $\boldsymbol{x}(U)=M$ by

$$
\operatorname{Ev}_{M}=\left\{\boldsymbol{x}(u)+\frac{1}{\kappa(u)} \boldsymbol{n}(u) \mid \kappa(u) \text { is a principal curvature at } p=\boldsymbol{x}(u), u \in U\right\}
$$

and the set of parallels of $\boldsymbol{x}(U)=M$ by

$$
P_{M}=\{\boldsymbol{x}(u)+r \cdot \boldsymbol{n}(u) \mid r \in \mathbb{R} \backslash\{0\}, u \in U\} .
$$

We also define the smooth mapping $\operatorname{Ev}_{\kappa}: U \rightarrow \mathbb{R}^{n}$ and $P_{r}: U \rightarrow \mathbb{R}^{n}$ by

$$
\operatorname{Ev}_{\kappa}(u)=\boldsymbol{x}(u)+\frac{1}{\kappa(u)} \boldsymbol{n}(u), P_{r}(u)=\boldsymbol{x}(u)+r \cdot \boldsymbol{n}(u),
$$

where we fix a principal curvature $\kappa(u)$ on $U$ at $u$ with $\kappa(u) \neq 0$ and a real number $r \neq 0$.

We now define family of functions in order to describe the evolute and parallels of a hypersurface in $\mathbb{R}^{n}$. For the purpose, we need some concepts and results in the theory of unfoldings of function germs. Define

$$
D: U \times\left(\mathbb{R}^{n} \backslash M\right) \rightarrow \mathbb{R}
$$

by $D(u, \boldsymbol{v})=\|\boldsymbol{x}(u)-\boldsymbol{v}\|^{2}$ and

$$
\bar{D}: U \times\left(\mathbb{R}^{n} \backslash M\right) \times \mathbb{R}_{+} \rightarrow \mathbb{R}
$$

by $\bar{D}(u, \boldsymbol{v}, t)=\|\boldsymbol{x}(u)-\boldsymbol{v}\|^{2}-t$, where $\mathbb{R}_{+}$is the set of positive real numbers. We call $D$ a distance squared function and $\bar{D}$ a graphlike distance squared function on $M=\boldsymbol{x}(U)$. Define $d_{v}(u)=D(u, \boldsymbol{v})$ and $\bar{d}_{v}(u, t)=\bar{D}(u, \boldsymbol{v}, t)$.

The following proposition follows from direct calculations:

Proposition 5.1. Let $\boldsymbol{x}: U \rightarrow \mathbb{R}^{n}$ be a hypersurface. Then

(1) $\left(\partial d_{v} / \partial u_{i}\right)(u)=0(i=1, \ldots, n-1)$ if and only if there exists a real number $r \in \mathbb{R} \backslash\{0\}$ such that $\boldsymbol{v}=\boldsymbol{x}(u)+r \cdot \boldsymbol{n}(u)$.

(2) $\left(\partial d_{v} / \partial u_{i}\right)(u)=0(i=1, \ldots, n-1)$ and $\operatorname{det}\left(\mathcal{H}\left(d_{v}\right)(u)\right)=0$ if and only if $\boldsymbol{v}=$ $\boldsymbol{x}(u)+(1 / \kappa(u)) \cdot \boldsymbol{n}(u)$.

(3) $\bar{d}_{v}(u, t)=\left(\partial \bar{d}_{v} / \partial u_{i}\right)(u, t)=0(i=1, \ldots, n-1)$ if and only if $\boldsymbol{v}=\boldsymbol{x}(u) \pm \sqrt{t} \cdot \boldsymbol{n}(u)$. Here $\mathcal{H}\left(d_{v}\right)(u)$ is the hessian matrix of the function $d_{v}$ at $u$.

We can detect the catastrophe set and bifurcation set of distance squared function $D$ and the discriminant set of graphlike distance squared function $\bar{D}$ by Proposition 5.1.

$$
C(D)=\left\{(u, \boldsymbol{v}) \in U \times\left(\mathbb{R}^{n} \backslash M\right) \mid \boldsymbol{v}=\boldsymbol{x}(u)+r \cdot \boldsymbol{n}(u), r \in \mathbb{R} \backslash\{0\}\right\},
$$




$$
\mathcal{B}_{D}=\left\{\boldsymbol{v} \in \mathbb{R}^{n} \backslash M \mid \boldsymbol{v}=\boldsymbol{x}(u)+\frac{1}{\kappa(u)} \cdot \boldsymbol{n}(u), u \in U\right\}
$$

and

$$
D(\bar{D})=\left\{(\boldsymbol{v}, t) \in\left(\mathbb{R}^{n} \backslash M\right) \times \mathbb{R}_{+} \mid \boldsymbol{v}=\boldsymbol{x}(u) \pm \sqrt{t} \cdot \boldsymbol{n}(u), u \in U\right\} .
$$

We can naturally interpret the evolutes of a hypersurface as a caustic and parallels of a hypersurface as graphlike wave fronts (big wave front).

Proposition 5.2. For a hypersurface $\boldsymbol{x}: U \rightarrow \mathbb{R}^{n}$, the distance squared function $D$ : $U \times\left(\mathbb{R}^{n} \backslash M\right) \rightarrow \mathbb{R}$ is a Morse family of functions and the graphlike distance squared function $\bar{D}: U \times\left(\mathbb{R}^{n} \backslash M\right) \times \mathbb{R}_{+} \rightarrow \mathbb{R}$ is a graphlike Morse family of hypersurfaces.

Proof. For any $\boldsymbol{v}=\left(v_{1}, \ldots, v_{n}\right) \in \mathbb{R}^{n} \backslash M$, we have $D(u, \boldsymbol{v})=\sum_{i=1}^{n}\left(x_{i}(u)-v_{i}\right)^{2}$, where $\boldsymbol{x}(u)=\left(x_{1}(u), \ldots, x_{n}(u)\right)$. We shall prove that the mapping

$$
\Delta D=\left(\frac{\partial D}{\partial u_{1}}, \ldots, \frac{\partial D}{\partial u_{n-1}}\right)
$$

is a non-singular at any point. The Jacobian matrix of $\Delta D$ is given by

$$
\left(\begin{array}{cccccc}
A_{11}(u) & \cdots & A_{1(n-1)}(u) & -2 x_{1 u_{1}}(u) & \cdots & -2 x_{n u_{1}}(u) \\
\vdots & \ddots & \vdots & \vdots & \ddots & \vdots \\
A_{(n-1) 1}(u) & \cdots & A_{(n-1)(n-1)} & -2 x_{1 u_{n-1}}(u) & \cdots & -2 x_{n u_{n-1}}(u)
\end{array}\right),
$$

where $A_{i j}(u)=2\left(\left\langle\boldsymbol{x}_{u_{i} u_{j}}(u), \boldsymbol{x}(u)-\boldsymbol{v}\right\rangle+\left\langle\boldsymbol{x}_{u_{i}}(u), \boldsymbol{x}_{u_{j}}(u)\right\rangle\right)$ and $\langle$,$\rangle is the inner product of$ $\mathbb{R}^{n}$. Since $\boldsymbol{x}: U \rightarrow \mathbb{R}^{n}$ is an embedding, the rank of the matrix

$$
\left(\begin{array}{ccc}
x_{1 u_{1}}(u) & \cdots & x_{n u_{1}}(u) \\
\vdots & \ddots & \vdots \\
x_{1 u_{n-1}}(u) & \cdots & x_{n u_{n-1}}(u)
\end{array}\right)
$$

is $n-1$ at any $u \in U$ and hence $D$ is a Morse family of functions. By definition, $\bar{D}$ is a graphlike Morse family of hypersurfaces.

By the method for constructing the Lagrangian submanifold germ from a Morse family of functions (cf. §2), we can define a Lagrangian submanifold germ whose generating family is the distance squared function $D$ of $M=\boldsymbol{x}(U)$ as follows: For a hypersurface $\boldsymbol{x}: U \rightarrow \mathbb{R}^{n}$ where $\boldsymbol{x}(u)=\left(x_{1}(u), \ldots, x_{n}(u)\right)$, we define

$$
L(D): C(D) \rightarrow T^{*} \mathbb{R}^{n}
$$

by

$$
L(D)(u, \boldsymbol{v})=\left(\boldsymbol{v},-2\left(x_{1}(u)-v_{1}\right), \ldots,-2\left(x_{n}(u)-v_{n}\right)\right),
$$

where $\boldsymbol{v}=\left(v_{1}, \ldots, v_{n}\right)$.

On the other hand, by the method for constructing the big Lagrangian submanifold germ from a big Morse family of functions (cf. §3), we can define a big Legendrian submanifold germ whose generating family is the graphlike distance squared function $\bar{D}$ of $M=\boldsymbol{x}(U)$. However, since $\bar{D}$ is a graphlike generating family, we can construct the graphlike Legendrian unfolding as follows: For a hypersurface $\boldsymbol{x}: U \rightarrow \mathbb{R}^{n}$ where 
$\boldsymbol{x}(u)=\left(x_{1}(u), \ldots, x_{n}(u)\right)$, we define

$$
\mathfrak{L}_{D}: C(D) \rightarrow J^{1}\left(\mathbb{R}^{n}, \mathbb{R}\right)
$$

by

$$
\mathfrak{L}_{D}(u, \boldsymbol{v})=\left(\boldsymbol{v},\|\boldsymbol{x}(u)-\boldsymbol{v}\|^{2},-2\left(x_{1}(u)-v_{1}\right), \ldots,-2\left(x_{n}(u)-v_{n}\right)\right),
$$

where $\boldsymbol{v}=\left(v_{1}, \ldots, v_{n}\right)$.

COROLlary 5.3. Under the above notations, $L(D)(C(D))$ is a Lagrangian submanifold such that the distance squared function $D$ is a generating family of $L(D)(C(D)$ ) and $\mathfrak{L}_{D}(C(D))$ is a graphlike Legendrian unfolding such that the graphlike distance squared function $\bar{D}$ is a graphlike generating family of $\mathfrak{L}_{D}(C(D))$.

\section{Contact with osculating hypersphere and families of tangent hyperspheres.} For a hypersurface $\boldsymbol{x}: U \rightarrow \mathbb{R}^{n}$, we consider the function $\mathcal{D}: \mathbb{R}^{n} \times\left(\mathbb{R}^{n} \backslash M\right) \rightarrow \mathbb{R}$ given by $\mathcal{D}(\boldsymbol{x}, \boldsymbol{v})=\|\boldsymbol{x}-\boldsymbol{v}\|^{2}$. We denote $\mathfrak{d}_{v}(\boldsymbol{x})=\mathcal{D}(\boldsymbol{x}, \boldsymbol{v})$ and we have a hypersphere $\mathfrak{d}_{v}{ }^{-1}\left(r^{2}\right)=S^{n-1}(\boldsymbol{v},|r|)$, where $S^{n-1}(\boldsymbol{v},|r|)$ is the hypersphere in $\mathbb{R}^{n}$ with center $\boldsymbol{v}$ and radius $|r|$. It is easy to show that $\mathfrak{d}_{v}$ is a submersion germ. For any $u \in U$, we consider a parallel point $\boldsymbol{v}=P_{r}(u)=\boldsymbol{x}(u)+r \cdot \boldsymbol{n}(u)$. Then we have

$$
\mathfrak{d}_{v} \circ \boldsymbol{x}(u)=\mathcal{D} \circ\left(\boldsymbol{x} \times i d_{\mathbb{R}^{n}}\right)(u, \boldsymbol{v})=r^{2}
$$

and

$$
\frac{\partial\left(\mathfrak{d}_{v} \circ \boldsymbol{x}\right)}{\partial u_{i}}(u)=\frac{\partial D}{\partial u_{i}}(u, \boldsymbol{v})=0,
$$

for $i=1, \ldots, n-1$ by Proposition 5.1. This means that the hypersphere $\mathfrak{d}_{v}^{-1}\left(r^{2}\right)=$ $S^{n-1}(\boldsymbol{v},|r|)$ is tangent to $M=\boldsymbol{x}(U)$ at $p=\boldsymbol{x}(u)$. In this case, we call $S^{n-1}(\boldsymbol{v},|r|)$ a tangent hypersphere at $p=\boldsymbol{x}(u)$ with center $\boldsymbol{v}$. However, there are infinitely many tangent hyperspheres at a general point depending on the real number $r$. If $\boldsymbol{v}$ is a point of the evolute, $\boldsymbol{v}=\operatorname{Ev}_{\kappa}(u)$, the tangent hypersphere with the center $\boldsymbol{v}$ is called the osculating hypersphere at $p=\boldsymbol{x}(u)$ which is uniquely determined.

Therefore for a parallel point $\boldsymbol{v}=P_{v}(u)=\boldsymbol{x}(u)+r \cdot \boldsymbol{n}(u)$, we have a regular foliation

$$
\mathcal{F}\left(\mathfrak{d}_{v}\right)=\left\{S^{n-1}(\boldsymbol{v}, \sqrt{c}) \mid c \in\left(\mathbb{R}_{+}, r^{2}\right)\right\}
$$

whose leaves are hyperspheres with center $\boldsymbol{v}$ such that the case $c=r^{2}$ corresponding to the tangent hypersphere with radius $|r|$. Moreover if $r=1 / \kappa(u)$, then $S^{n-1}(\boldsymbol{v},|1 / \kappa(u)|)$ is the osculating hypersphere. In this case, $\left(\boldsymbol{x}^{-1}\left(\mathcal{F}\left(\mathfrak{d}_{v}\right)\right), u\right)$ is a singular foliation germ at $u$ and we say that a osculating hyperspherical foliation of $M=\boldsymbol{x}(U)$ at $p=\boldsymbol{x}(u)$ (or, $u)$. We denote it by $\mathcal{O} \mathcal{F}(\boldsymbol{x}(U), u)$.

We shall consider the contact of hypersurfaces with osculating hypersphere. Let $\boldsymbol{x}_{i}:\left(U, u_{i}\right) \rightarrow\left(\mathbb{R}^{n}, p_{i}\right)(i=1,2)$ be hypersurface germs. Consider the distance squared functions $D_{i}:\left(U \times\left(\mathbb{R}^{n} \backslash M_{i}\right),\left(u_{i}, \boldsymbol{v}_{i}\right)\right) \rightarrow \mathbb{R}$ of $\boldsymbol{x}_{i}(U)=M_{i}$, where $\boldsymbol{v}_{i}=\operatorname{Ev}_{\kappa_{i}}\left(u_{i}\right)$. We denote that $d_{i, v_{i}}(u)=D_{i}\left(u, \boldsymbol{v}_{i}\right)$, then we have $d_{i, v_{i}}(u)=\mathfrak{d}_{v_{i}} \circ \boldsymbol{x}_{i}(u)$. By using Propositions 7.3 and 7.4 in Appendix, we have the following theorem:

THEOREM 6.1. Let $\boldsymbol{x}_{i}:\left(U, u_{i}\right) \rightarrow\left(\mathbb{R}^{n}, p_{i}\right)(i=1,2)$ be hypersurface germs such that the corresponding Lagrangian submanifold germs $L\left(D_{i}\right):\left(C\left(D_{i}\right),\left(u_{i}, \boldsymbol{v}_{i}\right)\right) \rightarrow T^{*} \mathbb{R}^{n}$ are 
Lagrange stable, where $\boldsymbol{v}_{i}=\operatorname{Ev}_{\kappa_{i}}\left(u_{i}\right)$ are centers of the osculating hyperspheres at $p_{i}$ respectively. Then the following conditions are equivalent:

(1) $K\left(\boldsymbol{x}_{1}(U), \mathcal{F}\left(\mathfrak{d}_{v_{1}}\right) ; p_{1}\right)=K\left(\boldsymbol{x}_{2}(U), \mathcal{F}\left(\mathfrak{d}_{v_{2}}\right) ; p_{2}\right)$

(2) $d_{1, v_{1}}$ and $d_{2, v_{2}}$ are $\mathcal{R}^{+}$-equivalent.

(3) $D_{1}$ and $D_{2}$ are $P-\mathcal{R}^{+}$-equivalent.

(4) $L\left(D_{1}\right)\left(C\left(D_{1}\right)\right)$ and $L\left(D_{2}\right)\left(C\left(D_{2}\right)\right)$ are Lagendrian equivalent.

(5) (a) The rank and signature of the $\mathcal{H}\left(d_{1, v_{1}}\right)\left(u_{1}\right)$ and $\mathcal{H}\left(d_{2, v_{2}}\right)\left(u_{2}\right)$ are equal, and

(b) There is an isomorphism $\gamma: \mathcal{R}_{2}\left(d_{1, v_{1}}\right) \rightarrow \mathcal{R}_{2}\left(d_{2, v_{2}}\right)$ such that $\gamma\left(\left[d_{1, v_{1}}\right]\right)=$ $\left[d_{2, v_{2}}\right]$.

Proof. By Proposition 7.3, the condition (1) is equivalent to the condition (2). Since both of $L\left(D_{i}\right)\left(C\left(D_{i}\right)\right)$ are Lagrange stable, both of $D_{i}$ are $\mathcal{R}^{+}$-versal deformation of $d_{i, v_{i}}$. By the uniqueness theorem on the $\mathcal{R}^{+}$-versal deformation of a function, (2) is equivalent to (3). By Theorem 2.2, (3) is equivalent to (4). It also follows from Theorem 2.2 that both of $d_{i, v_{i}}$ satisfy the Milnor condition. Therefore we can apply Proposition 7.4 to our situation, so that (2) is equivalent to (5). This completes the proof.

On the other hand, for a hypersurface $\boldsymbol{x}: U \rightarrow \mathbb{R}^{n}$, we consider the function $\overline{\mathcal{D}}$ : $\mathbb{R}^{n} \times\left(\mathbb{R}^{n} \backslash M\right) \times \mathbb{R}_{+} \rightarrow \mathbb{R}$ given by $\overline{\mathcal{D}}(\boldsymbol{x}, \boldsymbol{v}, t)=\|\boldsymbol{x}-\boldsymbol{v}\|^{2}-t$. We define $\overline{\mathfrak{d}}_{v}(\boldsymbol{x}, t)=\overline{\mathcal{D}}(\boldsymbol{x}, \boldsymbol{v}, t)$. It is easy to show that $\overline{\mathfrak{d}}_{v, t}$ is a submersion germ for each $t \in \mathbb{R}_{+}$. Then for any $u \in U$ and the parallel point $\boldsymbol{v}=P_{ \pm \sqrt{t}}(u)=\boldsymbol{x}(u) \pm \sqrt{t} \cdot \boldsymbol{n}(u)$, we have

$$
\overline{\mathfrak{d}}_{v} \circ\left(\boldsymbol{x} \times i d_{\mathbb{R}_{+}}\right)(u, t)=0
$$

and

$$
\frac{\partial\left(\overline{\mathfrak{d}}_{v} \circ\left(\boldsymbol{x} \times i d_{\mathbb{R}_{+}}\right)\right)}{\partial u_{i}}(u, t)=\frac{\partial \bar{D}}{\partial u_{i}}(u, \boldsymbol{v}, t)=0,
$$

for $i=1, \ldots, n-1$ by Proposition 5.1. Hence we define a one-parameter family of tangent hyperspheres

$$
\mathcal{H S}(\boldsymbol{v})=\left(\overline{\mathfrak{d}}_{v}\right)^{-1}(0)=\left\{(\boldsymbol{x}, t) \in \mathbb{R}^{n} \times \mathbb{R}_{+} \mid \boldsymbol{x} \in S^{n-1}(\boldsymbol{v}, \sqrt{t})\right\} .
$$

We call $\boldsymbol{v}$ the center of the one-parameter family of tangent hyperspheres at $(\boldsymbol{x}(u), t)$. By using Theorem 7.2 in Appendix, we have the following theorem:

THEOREM 6.2. Let $\boldsymbol{x}_{i}:\left(U, u_{i}\right) \rightarrow\left(\mathbb{R}^{n}, p_{i}\right)(i=1,2)$ be hypersurface germs such that the corresponding graphlike Legrangian unfoldings $\mathfrak{L}_{D_{i}}:\left(C\left(D_{i}\right),\left(u_{i}, \boldsymbol{v}_{i}\right)\right) \rightarrow J^{1}\left(\mathbb{R}^{n}, \mathbb{R}\right)$ are S.P $P^{+}$-Legendre stable, where $\boldsymbol{v}_{i}$ are centers of one-parameter family of tangent hyperspheres at $\left(p_{i}, t_{i}\right)$. Then the following conditions are equivalent:

$\left(1^{\prime}\right) \operatorname{PK}\left(\boldsymbol{x}_{1}(U), \mathcal{H S}\left(\boldsymbol{v}_{1}\right) ;\left(p_{1}, t_{1}\right)\right)=\operatorname{PK}\left(\boldsymbol{x}_{2}(U), \mathcal{H S}\left(\boldsymbol{v}_{2}\right) ;\left(p_{2}, t_{2}\right)\right)$.

$\left(2^{\prime}\right) \bar{d}_{1, v_{1}}$ and $\bar{d}_{2, v_{2}}$ are S.P-K- $\mathcal{K}$-equivalent.

$\left(3^{\prime}\right) \bar{D}_{1}$ and $\bar{D}_{2}$ are $\boldsymbol{v}$-S.P $P^{+}-\mathcal{K}$-equivalent.

$\left(4^{\prime}\right) \mathfrak{L}_{D_{1}}\left(C\left(D_{1}\right)\right)$ and $\mathfrak{L}_{D_{2}}\left(C\left(D_{2}\right)\right)$ are $S . P^{+}$-Legendrian equivalent.

$\left(5^{\prime}\right)$ The graphlike wave fronts $W\left(\mathfrak{L}_{D_{1}}\right)$ and $W\left(\mathfrak{L}_{D_{2}}\right)$ are S.P $P^{+}$-diffeomorphic.

Proof. By Theorem 7.2, the condition $\left(1^{\prime}\right)$ is equivalent to the condition $\left(2^{\prime}\right)$. Since both of $\mathfrak{L}_{D_{i}}\left(C\left(D_{i}\right)\right)$ are $S . P^{+}$-Legendre stable, both of $\bar{D}_{i}$ are $S . P^{+}-\mathcal{K}$-versal deformation of $\bar{d}_{i, v_{i}}$. By Proposition 3.2, $\left(2^{\prime}\right)$ is equivalent to $\left(3^{\prime}\right)$. By Theorem 3.3, $\left(3^{\prime}\right)$ is equivalent to 
$\left(4^{\prime}\right)$. Also by the assumption that $\mathfrak{L}_{D_{i}}\left(C\left(D_{i}\right)\right)$ are $S P^{+}$-Legendre stable, the assumption of Proposition 3.5 is satisfied for $\mathfrak{L}_{D_{i}}$. It follows that $\left(4^{\prime}\right)$ is equivalent to $\left(5^{\prime}\right)$. This completes the proof.

By Propositions 4.2 and 4.4, two Lagrangian submanifold germs are Lagrangian equivalent if and only if the induced graphlike Legendrian unfolding germs are $S P^{+}$Legendrian equivalent under the condition of Lagrange stability. Moreover, by Proposition 4.1 , if $\mathfrak{L}_{D}:(C(D),(u, \boldsymbol{v})) \rightarrow J^{1}\left(\mathbb{R}^{n}, \mathbb{R}\right)$ is $S . P^{+}$-Legendre stable, then $L(D)$ : $(C(D),(u, \boldsymbol{v})) \rightarrow T^{*} \mathbb{R}^{n}$ is a Lagrange stable. Then we have the following corollary of Theorems 6.1 and 6.2:

COROllary 6.3. Let $\boldsymbol{x}_{i}:\left(U, u_{i}\right) \rightarrow\left(\mathbb{R}^{n}, p_{i}\right)(i=1,2)$ be hypersurface germs such that the corresponding graphlike Legrangian unfoldings $\mathfrak{L}_{D_{i}}:\left(C\left(D_{i}\right),\left(u_{i}, \boldsymbol{v}_{i}\right)\right) \rightarrow J^{1}\left(\mathbb{R}^{n}, \mathbb{R}\right)$ are S.P $P^{+}$-Legendre stable, where $\boldsymbol{v}_{i}=\operatorname{Ev}_{\kappa_{i}}\left(u_{i}\right)$ are centers of the osculating hyperspheres of $\boldsymbol{x}_{i}(U)$ respectively. Then the following conditions are equivalent:

(1) $K\left(\boldsymbol{x}_{1}(U), \mathcal{F}\left(\mathfrak{d}_{v_{1}}\right) ; p_{1}\right)=K\left(\boldsymbol{x}_{2}(U), \mathcal{F}\left(\mathfrak{d}_{v_{2}}\right) ; p_{2}\right)$.

(2) $d_{1, v_{1}}$ and $d_{2, v_{2}}$ are $\mathcal{R}^{+}$-equivalent.

(3) $D_{1}$ and $D_{2}$ are $P-\mathcal{R}^{+}$-equivalent.

(4) $L\left(D_{1}\right)\left(C\left(D_{1}\right)\right)$ and $L\left(D_{2}\right)\left(C\left(D_{2}\right)\right)$ are Lagrangian equivalent.

(5) (a) The rank and signature of the $\mathcal{H}\left(d_{1, v_{1}}\right)\left(u_{1}\right)$ and $\mathcal{H}\left(d_{2, v_{2}}\right)\left(u_{2}\right)$ are equal, and

(b) There is an isomorphism $\gamma: \mathcal{R}_{2}\left(d_{1, v_{1}}\right) \rightarrow \mathcal{R}_{2}\left(d_{2, v_{2}}\right)$ such that $\gamma\left(\left[d_{1, v_{1}}\right]\right)=$ $\left[d_{2, v_{2}}\right]$.

(6) $P K\left(\boldsymbol{x}_{1}(U), \mathcal{H S}\left(\boldsymbol{v}_{1}\right) ;\left(p_{1}, 1 / \kappa_{1}\left(u_{1}\right)\right)\right)=P K\left(\boldsymbol{x}_{2}(U), \mathcal{H} \mathcal{S}\left(\boldsymbol{v}_{2}\right) ;\left(p_{2}, 1 / \kappa_{2}\left(u_{2}\right)\right)\right)$.

(7) $\bar{d}_{1, v_{1}}$ and $\bar{d}_{2, v_{2}}$ are S.P-K-equivalent.

(8) $\bar{D}_{1}$ and $\bar{D}_{2}$ are $\boldsymbol{v}$-S.P. ${ }^{+}{ }_{-} \mathcal{K}$-equivalent.

(9) $\mathfrak{L}_{D_{1}}\left(C\left(D_{1}\right)\right)$ and $\mathfrak{L}_{D_{2}}\left(C\left(D_{2}\right)\right)$ are $S . P^{+}$-Legendrian equivalent.

(10) The graphlike wave fronts $W\left(\mathfrak{L}_{D_{1}}\right)$ and $W\left(\mathfrak{L}_{D_{2}}\right)$ are S.P ${ }^{+}$-diffeomorphic.

More precisely, we can replace the condition that $\mathfrak{L}_{D_{i}}:\left(C\left(D_{i}\right),\left(u_{i}, \boldsymbol{v}_{i}\right)\right) \rightarrow J^{1}\left(\mathbb{R}^{n}, \mathbb{R}\right)$ are $S . P^{+}$-Legendre stable with the condition $L\left(D_{i}\right):\left(C\left(D_{i}\right),\left(u_{i}, \boldsymbol{v}_{i}\right)\right) \rightarrow T^{*} \mathbb{R}^{n}$ are Lagrange stable by Theorem 4.6. As a consequence, we have the following result:

THEOREM 6.4. Let $\boldsymbol{x}_{i}:\left(U, u_{i}\right) \rightarrow\left(\mathbb{R}^{n}, p_{i}\right)(i=1,2)$ be hypersurface germs such that the corresponding Lagrangian submanifold germs $L\left(D_{i}\right):\left(C\left(D_{i}\right),\left(u_{i}, \boldsymbol{v}_{i}\right)\right) \rightarrow T^{*} \mathbb{R}^{n}$ are Lagrange stable, where $\boldsymbol{v}_{i}=\operatorname{Ev}_{\kappa_{i}}\left(u_{i}\right)$ are centers of the osculating hyperspheres of $\boldsymbol{x}_{i}(U)$ respectively. Then conditions (1) to (10) in Corollary 6.3 are also equivalent.

7. Appendix. We start by recalling the theory of contact with submanifolds due to Montaldi [18]. Let $X_{i}, Y_{i}(i=1,2)$ be submanifolds of $\mathbb{R}^{n}$ with $\operatorname{dim} X_{1}=\operatorname{dim} X_{2}$ and $\operatorname{dim} Y_{1}=\operatorname{dim} Y_{2}$. We say that the contact of $X_{1}$ and $Y_{1}$ at $y_{1}$ is the same type as the contact of $X_{2}$ and $Y_{2}$ at $y_{2}$ if there is a diffeomorphism germ $\Phi:\left(\mathbb{R}^{n}, y_{1}\right) \rightarrow\left(\mathbb{R}^{n}, y_{2}\right)$ such that $\Phi\left(X_{1}\right)=X_{2}$ and $\Phi\left(Y_{1}\right)=Y_{2}$. In this case we write $K\left(X_{1}, Y_{1} ; y_{1}\right)=K\left(X_{2}, Y_{2} ; y_{2}\right)$. It is clear that in the definition $\mathbb{R}^{n}$ could be replaced by any manifold. In his paper [18], Montaldi gives a characterization of the notion of contact by using the terminology of singularity theory. 
Theorem 7.1. Let $X_{i}(i=1,2)$ be submanifolds of $\mathbb{R}^{n}$ with $\operatorname{dim} X_{1}=\operatorname{dim} X_{2}$. Let $g_{i}:\left(X_{i}, x_{i}\right) \rightarrow\left(\mathbb{R}^{n}, y_{i}\right)$ be immersion germs and $f_{i}:\left(\mathbb{R}^{n}, y_{i}\right) \rightarrow\left(\mathbb{R}^{p}, 0\right)$ be submersion germs with $\left(Y_{i}, y_{i}\right)=\left(f_{i}^{-1}(0), y_{i}\right)$. Then $K\left(X_{1}, Y_{1} ; y_{1}\right)=K\left(X_{2}, Y_{2} ; y_{2}\right)$ if and only if $f_{1} \circ g_{1}$ and $f_{2} \circ g_{2}$ are $\mathcal{K}$-equivalent. For the definition of $\mathcal{K}$-equivalence, see [17].

For our purpose this theorem is not sufficient. We need the theory of contact of submanifolds with one-parameter families of hypersurfaces. We have two kinds of theories which describe the contact with one-parameter families of hypersurfaces.

Firstly we consider the one-parameter families of hypersurfaces. Let $X_{i}(i=1,2)$ be submanifolds in $\mathbb{R}^{n}$ with $\operatorname{dim} X_{1}=\operatorname{dim} X_{2}$ and $f_{i}:\left(\mathbb{R}^{n} \times \mathbb{R},\left(y_{i}, t_{i}\right)\right) \rightarrow(\mathbb{R}, 0)$ be function germs such that $f_{i, t}$ is a submersion germ for each $t \in\left(\mathbb{R}, t_{i}\right)$. Here, we define $f_{i, t}(y)=f_{i}(y, t)$. We have hypersurface germs $\left(\mathbb{R}^{n} \times \mathbb{R},\left(y_{i}, t_{i}\right)\right) \supset \mathcal{Y}\left(f_{i}\right)=f_{i}^{-1}(0)$. We say that the parametrized contact of $X_{1}$ and $\mathcal{Y}_{1}$ at $\left(y_{1}, t_{1}\right)$ is the same type as the parametrized contact of $X_{2}$ and $\mathcal{Y}_{2}$ at $\left(y_{2}, t_{2}\right)$ if there is a diffeomorphism germ $\Phi:\left(\mathbb{R}^{n} \times \mathbb{R},\left(y_{1}, t_{1}\right)\right) \rightarrow$ $\left(\mathbb{R}^{n} \times \mathbb{R},\left(y_{2}, t_{2}\right)\right)$ with the form $\Phi(y, t)=\left(\phi(y, t), t+\left(t_{2}-t_{1}\right)\right)$ such that $\Phi\left(X_{1} \times \mathbb{R}\right)=X_{2} \times \mathbb{R}$ and $\Phi\left(\mathcal{Y}_{1}\right)=\mathcal{Y}_{2}$. In this case we write

$$
P K\left(X_{1}, \mathcal{Y}_{1} ;\left(y_{1}, t_{1}\right)\right)=\operatorname{PK}\left(X_{2}, \mathcal{Y}_{2} ;\left(y_{2}, t_{2}\right)\right) .
$$

We can show the following parametric version of Montaldi's theorem just along the line of the proof of the original theorem of Montaldi [18].

THEOREM 7.2. Under the above notations, $\operatorname{PK}\left(X_{1}, \mathcal{Y}_{1} ;\left(y_{1}, t_{1}\right)\right)=P K\left(X_{2}, \mathcal{Y}_{2} ;\left(y_{2}, t_{2}\right)\right)$ if and only if $f_{1} \circ\left(g_{1} \times i d_{\mathbb{R}}\right)$ and $f_{2} \circ\left(g_{2} \times i d_{\mathbb{R}}\right)$ are S.P-K-K-equivalent.

Secondary we consider the codimension one foliation germs. Let $X_{i}(i=1,2)$ be submanifolds of $\mathbb{R}^{n}$ with $\operatorname{dim} X_{1}=\operatorname{dim} X_{2}, g_{i}:\left(X_{i}, \bar{x}_{i}\right) \rightarrow\left(\mathbb{R}^{n}, \bar{y}_{i}\right)$ be immersion germs and $f_{i}:\left(\mathbb{R}^{n}, \bar{y}_{i}\right) \rightarrow(\mathbb{R}, 0)$ be submersion germs. For a submersion germ $f:\left(\mathbb{R}^{n}, 0\right) \rightarrow$ $(\mathbb{R}, 0)$, we define $\mathcal{F}(f)$ to be the regular foliation defined by $f$; i.e., $\mathcal{F}(f)=\left\{f^{-1}(c) \mid c \in\right.$ $(\mathbb{R}, 0)\}$. We say that the contact of $X_{1}$ with the regular foliation $\mathcal{F}\left(f_{1}\right)$ at $\bar{y}_{1}$ is the same type as the contact of $X_{2}$ with the regular foliation $\mathcal{F}\left(f_{2}\right)$ at $\bar{y}_{2}$ if there is a diffeomorphism germ $\Phi:\left(\mathbb{R}^{n}, \bar{y}_{1}\right) \rightarrow\left(\mathbb{R}^{n}, \bar{y}_{2}\right)$ such that $\Phi\left(X_{1}\right)=X_{2}$ and $\Phi\left(Y_{1}(c)\right)=Y_{2}(c)$ for each $c \in$ $(\mathbb{R}, 0)$, where $Y_{i}(c)=f_{i}^{-1}(c)$. In this case we write $K\left(X_{1}, \mathcal{F}\left(f_{1}\right) ; \bar{y}_{1}\right)=K\left(X_{2}, \mathcal{F}\left(f_{2}\right) ; \bar{y}_{2}\right)$. We apply the method of Goryunov [7] to the case of $\mathcal{R}^{+}$-equivalence among function germs, so that we have the following:

Proposition 7.3 ([7, Appendix $])$. Let $X_{i}(i=1,2)$ be submanifolds of $\mathbb{R}^{n}$ with $\operatorname{dim} X_{1}=$ $\operatorname{dim} X_{2}-1$ (i.e., hypersurface), $g_{i}:\left(X_{i}, \bar{x}_{i}\right) \rightarrow\left(\mathbb{R}^{n}, \bar{y}_{i}\right)$ be immersion germs and $f_{i}:$ $\left(\mathbb{R}^{n}, \bar{y}_{i}\right) \rightarrow(\mathbb{R}, 0)$ be submersion germs. We assume that $\bar{x}_{i}$ are singularities of function germs $f_{i} \circ g_{i}:\left(X_{i}, \bar{x}_{i}\right) \rightarrow(\mathbb{R}, 0)$. Then $K\left(X_{1}, \mathcal{F}\left(f_{1}\right) ; \bar{y}_{1}\right)=K\left(X_{2}, \mathcal{F}\left(f_{2}\right) ; \bar{y}_{2}\right)$ if and only if $f_{1} \circ g_{1}$ and $f_{2} \circ g_{2}$ are $\mathcal{R}^{+}$-equivalent.

On the other hand, Golubitsky and Guillemin [6] have given an algebraic characterization of $\mathcal{R}^{+}$-equivalence among function germs. We denote by $C_{0}^{\infty}(X)$ the set of function germs $(X, 0) \rightarrow \mathbb{R}$. Let $J_{f}$ be the Jacobian ideal in $C_{0}^{\infty}(X)$ (i.e., $J_{f}=$ $\left.\left\langle\partial f / \partial x_{1}, \ldots, \partial f / \partial x_{n}\right\rangle_{C_{0}^{\infty}(X)}\right)$. Let $\mathcal{R}_{k}(f)=C_{0}^{\infty}(X) / J_{f}^{k}$ and $[f]$ be the image of $f$ in this local ring. We say that $f$ satisfies the Milnor condition if $\operatorname{dim}_{\mathbb{R}} \mathcal{R}_{1}(f)<\infty$. 
Proposition 7.4 ([6, Proposition 4.1]). Let $f$ and $g$ be germs of functions at 0 in $X$ satisfying the Milnor condition with $d f(0)=d g(0)=0$. Then $f$ and $g$ are $\mathcal{R}^{+}$-equivalent if

(1) The rank and signature of the Hessians $\mathcal{H}(f)(0)$ and $\mathcal{H}(g)(0)$ are equal, and

(2) There is an isomorphism $\gamma: \mathcal{R}_{2}(f) \rightarrow \mathcal{R}_{2}(g)$ such that $\gamma([f])=[g]$.

\section{References}

[1] V. I. Arnol'd, S. M. Gusein-Zade and A. N. Varchenko, Singularities of Differentiable Maps, Vol. I, Birkhäuser, 1986.

[2] V. I. Arnol'd, Singularities of Caustics and Wave Fronts, Mathematics and its Applications 62, Kluwer Academic Publishers, 1990.

[3] J. W. Bruce, Wavefronts and parallels in Euclidean space, Math. Proc. Camb. Phil. Soc. 93 (1983), 323-333.

[4] J. Damon, The unfolding and determinacy theorems for subgroups of $\mathcal{A}$ and $\mathcal{K}$, Memoirs of Amer. Math. Soc. 50 (1984), no. 306.

[5] J. Ehlers and E. T. Newman, The theory of caustics and wave front singularities with physical applications, J. Math. Physics 41 (2000), 3344-3378.

[6] M. Golubitsky and V. Guillemin, Contact equivalence for Lagrangian manifolds, Adv. Math. 15 (1975), 375-387.

[7] V. V. Goryunov, Projections of generic surfaces with boundaries, Adv. Soviet Math. 1 (1990), 157-200.

[8] W. Hasse, M. Kriele and V. Perlick, Caustics of wavefronts in general relativity, Class. Quantum Grav. 13 (1996), 1161-1182.

[9] S. Izumiya, Perestroikas of optical wave fronts and graphlike Legendrian unfoldings, J. Differential Geom. 38 (1993), 485-500.

[10] S. Izumiya, Completely integrable holonomic systems of first-order differential equations, Proc. Roy. Soc. Edinburgh 125A (1995), 567-586.

[11] S. Izumiya, Differential Geometry from the viewpoint of Lagrangian or Legendrian singularity theory, in: Singularity Theory (Marseille, 2005), D. Chéniot et al. (eds.), World Scientific, 2007, 241-275.

[12] S. Izumiya and Y. Kurokawa, Holonomic systems of Clairaut type, Differential Geom. Appl. 5 (1995), 219-235.

[13] S. Izumiya, D.-H. Pei and M. Takahashi, Singularities of evolutes of hypersurfaces in hyperbolic space, Proc. Edinburgh Math. Soc. 47 (2004), 131-153.

[14] S. Izumiya, D.-H. Pei, M. C. Romero Fuster and M. Takahashi, The horospherical geometry of submanifolds in hyperbolic n-space, J. London Math. Soc. 71 (2005), 779-800.

[15] S. Izumiya and M. Takahashi, Spacelike parallels and evolutes in Minkowski pseudospheres, J. of Geometry and Physics 57 (2007), 1569-1600.

[16] M. Kokubu, W. Rossman, K. Saji, M. Umehara and K. Yamada, Singularities of flat fronts in hyperbolic space, Pacific J. Math. 221 (2005), 303-351.

[17] J. Martinet, Singularities of Smooth Functions and Maps, London Math. Soc. Lecture Note Series 58, Cambridge Univ. Press, 1982.

[18] J. A. Montaldi, On contact between submanifolds, Michigan Math. J. 33 (1986), 195-199.

[19] I. Porteous, The normal singularities of a submanifold, J. Diff. Geom. 5 (1971), 543-564. 
[20] M. Takahashi, Holonomic systems of general Clairaut type, Hokkaido Math. J. 34 (2005), 247-263.

[21] G. Wassermann, Stability of caustics, Math. Ann. 216 (1975), 43-50.

[22] V. M. Zakalyukin, Reconstructions of fronts and caustics depending on a parameter and versality of mappings, J. Soviet Math. 27 (1983), 2713-2735.

[23] V. M. Zakalyukin, Envelope of families of wave fronts and control theory, Proc. Steklov Inst. Math. 209 (1995), 114-123. 